\title{
CircRNAs: Insights into Gastric Cancer
}

\author{
Sanjana Rajgopal Sherine Joanna Fredrick Venkatachalam Deepa Parvathi \\ Department of Biomedical Sciences, Sri Ramachandra Institute of Higher Education and Research, Chennai, India
}

\section{Keywords}

Circular RNAs · Gastric cancer · Biomarker · Diagnosis

\begin{abstract}
Background: Gastric cancer (GC) is recorded as the fifth most common cancer globally. The classic resemblance of early symptoms of chronic gastritis including nausea, dysphagia, and dyspepsia with GC is the current challenge limiting the early diagnosis of GC. The current diagnostic procedures of GC are limited due to their invasive nature. This directs the research question toward alternative approaches, specifically at the molecular level. Recent advances in molecular regulation of cancer suggest the prominence of circular RNAs (circRNAs) in the multistep process of tumourigenesis. Summary: CircRNAs are a class of non-coding RNAs, abundant in eukaryotes, with key roles in regulating genes and miRNAs as well as the alteration of processes involved in pathological conditions. Research studies have demonstrated the participation of circRNAs in the initiation and progression of tumours. This review provides a comprehensive insight into the potential of circRNAs as disease biomarkers for the early detection and treatment of GC. Key Messages: This study is an amalgamation of the implications and future prospects of circRNAs for the detection and potential treatment of GC.
\end{abstract}

(C) 2021 The Author(s)

Published by S. Karger AG, Basel
(C) 2021 The Author(s)

Published by S. Karger AG, Basel

This is an Open Access article licensed under the Creative Commons Attribution-NonCommercial-4.0 International License (CC BY-NC) (http://www.karger.com/Services/OpenAccessLicense), applicable to the online version of the article only. Usage and distribution for commercial purposes requires written permission.

\section{Introduction}

Gastric cancer (GC) is the fifth most common malignancy worldwide and has the third highest mortality rate [1]. The late-onset nature of the symptoms and their similarity to those of chronic gastritis is a major contributing factor to its late diagnosis and high mortality rate. The limited understanding of the pathways associated with the disease directs the need for intensive research.

Over the previous decade, the functionality of noncoding RNAs (ncRNAs) has received a great deal of attention. The role of such molecules in regulation and signal transduction cannot be ignored. Studies have focused on discovering the relationship between ncRNAs and human diseases. Among these, the connection between circRNAs and cancer, particularly GC, has been explored with increasing fervour.

CircRNAs were discovered through hydrodynamic and thermodynamic analysis of isolated tomato RNA viroids [2]. The novel functions of circRNAs in gene regulation were elucidated by studies on the cerebellar degeneration-related protein 1 (CDR1) [3]. With high-throughput sequencing and bioinformatic tools, circRNA families have been established as facilitators of gene expression in human cells [4].

Molecular regulation is a crucial mediator of carcinogenesis, controlling a host of cascades involving genes and signalling molecules. The participation of circRNAs in the initiation and progression of tumours along with its contribution to early detection and treatment of GC is elaborated in this review.

Correspondence to:

Venkatachalam Deepa Parvathi, deepaparvathi@sriramachandra.edu.in 


\section{Biogenesis}

Previously, circRNAs were thought to be a splicing error of exon-skipping events [5]. They are synthesized through backsplicing of heteronuclear RNA, differing from the typical $5^{\prime}-3^{\prime}$ manner, catalysed by the spliceosomal machinery or by ribozymes [6]. The primary question in biogenesis was the method of circularization. The process can be represented by 2 major models of circularization; the lariat-mediated method and the intronpaired-mediated method. The former involves covalent binding between the $3^{\prime}$ splice donor and the $5^{\prime}$ splice acceptor [7] while the latter takes place by complementary base-pair binding between the intronic motifs flanking the circularized exon [8]. It is still uncertain as to which of these models is accurate or whether they occur simultaneously.

CircRNAs can be structurally classified as follows:

1. Exonic circRNAs (EcRNAs): they constitute an exoncontaining lariat, containing either single or multiple exons, finally spliced into an exon circle [7].

2. Exon-intron circRNAs (EIciRNAs): in case of retention of an intron between the exons, the transcript circularizes to form EIciRNAs [7].

3. Intronic circRNAs (ciRNAs): they involve binding of a 7-nucleotide GU-rich component proximal to the $5^{\prime}$ splice site and an 11-nucleotide C-rich component proximal to the branchpoint site during backsplicing [9].

EcRNAs are detected primarily in the cytoplasm [10], while ciRNAs and EIciRNAs are more prominent in the nucleus $[9,11]$. Synthesis of circRNAs involves a plethora of regulators, namely, intronic sequences, enzymes, and protein factors. Of these molecules, the most frequently observed are adenosine deaminase which acts on RNA [12] and RNA-binding proteins (RBPs), particularly Quaking protein [13] and muscleblind-like splicing regulator 1 [14]. Backsplicing is a naturally slow process due to the influence of premRNA splicing and distances between RBP-induced splicing sites. Other contributing factors include the activity of RNA rapid polymerase II, cis-acting elements, and feedback mechanisms [15]. The cellular concentration of circRNAs is dependent on the regulatory activity of these molecules.

A significant portion of intracellular circRNAs is scavenged by the extracellular vesicles (EVs). The EVs rupture to release circRNAs into the extracellular fluid, from where it is recruited to its designated pathway [16].

\section{Properties}

Characterization of circRNAs has taken prominence in biomolecular research owing to their unique structure and nature that stand out from other ncRNAs. With advancing RNA-sequencing technology, thousands of circRNAs have been identified in eukaryotes, and the mechanism of synthesis creates diversity in the circRNA population [17]. Due to their stability and a long half-life, circRNAs are nearly 10 times more abundant than linear RNAs in cells, extracellular fluid, and body fluids [18]. Evidence for the diverse nature of circRNAs was obtained in a study that detected 25,000 circRNAs in human fibroblasts alone [7].

CircRNAs are resistant to ribonucleases unlike linear RNAs [19]. Resistance to these enzymes is attributed to the covalently closed structure without $3^{\prime} / 5^{\prime}$ end polarity and a polynucleotide tail [20]. The expression patterns of circRNAs are highly conserved, observed through studies performed on plants, mice, zebrafish, and humans [21, 22].

The expression of circRNAs is known to be cell-type specific [23]. The specificity of circRNAs is particular to the tissue type and organism and at times even to different species as well. This aspect of circRNAs promotes its usage as a target of disease discovery and treatment. Extensive research has indicated that circRNAs mediate several molecular events in cancer such as the regulation of proliferation and metastasis [24]. Prominently, their stable expression in saliva, blood, and exosomes make them suitable biomarkers for studies in GC [20].

\section{Functions}

CircRNAs have the primary function of regulating gene expression by acting as miRNA sponges. As mentioned earlier, circRNAs are transported out of cells via EVs and acquired by other cells through the intake of the EVs, suggesting their contribution to cell signalling [25]. CircRNAs have the potential to be translated into functional proteins as a result of backsplicing $[4,20,26]$.

\section{MicroRNA Sponging}

CircRNAs function as miRNA sponges to sequester miRNA and prevent them from binding to target genes. Multiple target sites have been identified by bioinformatic prediction tools and high-throughput sequencing analysis of circRNA-miRNA interactions. For instance, RBP sites and the Argonaute protein family-binding site have been located on circRNAs [27-30]. 
The earliest identified functional feature of circRNAs was that of a microRNA (miR) sponge which sequesters and regulates the activity of specific miRs. The trailblazing example of cerebellar degeneration-associated protein 1 antisense transcript (CDRlas) showed that the molecule contains over $70 \mathrm{miR}$-7-binding domains which modulate the degradation of mRNA [30]. Such effects on this miR could thus lead to functional changes, such as the interaction of Cdrlas/miR-7 which was reported to affect $\beta$-cell function and enhance insulin secretion [31].

The multiple miR-binding sites observed on various other circRNAs such as CCDC66 suggest their ability to parallelly regulate various miRs. For instance, circHIPK3 binds to and inhibits the activity of miR-124 and also sequesters miR-379 which targets insulin-like growth factor 1 (IGF1) [32-34].

It is important to note that very few exceptional circRNAs, including the ones mentioned here, have documented proof of this unique function. Sponging capabilities could be attributed to circRNAs with higher expression levels [35], though experimental validation is needed via stoichiometric analysis.

CircRNAs have been shown to compete with mRNAs for miRNA binding in the cytoplasm [18]. CircRNAs act as competing endogenous RNA (ceRNA) which is relevant to signal transduction processes in tumour progression including cell proliferation, invasion, migration, and apoptosis. CircRNAs acting as ceRNAs absorb miRNA and competitively inhibit them, thus blocking miRNAs from regulating the expression of target genes.

This results in the alteration of cell function and growth $[32,36]$. Abundantly expressed circRNAs such as circHIPK3 have been found to regulate cell growth by sponging multiple miRNAs [32], and the circRNA HRCR has been found to sponge and thus inhibit miR-223, causing alterations in hypertrophic responses [37].

The involvement of circRNAs in signalling processes has been reported in other cancers. The circRNA CDR1as inhibits miRNA-7 (which acts as a tumour suppressor on EGFR and IGF-IR/PTEN/PI3K/AKT pathways), thus stimulating cell proliferation in colorectal cancer [38]. The expression of hsa_circ_0001982 has been reported to be increased in breast cancer tissue; bioinformatic prediction tools suggest that its target miRNA is miR-143 (which behaves as a tumour suppressor). The inhibition of miR143 by this circRNA was seen to alter cellular activity by promoting the proliferation and invasion of breast cancer cells [39]. CiRNAs and EIciRNAs (which retain some introns) can regulate alternative splicing, transcription, and the expression of parental genes [40].

CircRNAs: Insights into Gastric Cancer

\section{Transcription and Splicing Regulation}

The ciRNAs and EIciRNAs, abundantly present in the nucleus, play roles in the regulation of transcription of host genes [11]. The biogenesis of circRNAs during circularization is suggested to compete with the spliceosome machinery involved in the splicing process of pre-mRNA to linear RNA. Thus, as more circRNAs are generated, fewer linear mRNAs are generated; this can be attributed to the negative correlation between circRNA levels and splicing activity. The resulting mRNA also lacks exons as these exons are incorporated into the circRNAs. This form of exon skipping during circularization plays a significant role in regulating the expression of genes $[14,41$, 42].

Upon forming complexes with U1 spliceosomal RNA (U1 snRNP) and RNA polymerase II (Pol II), EIciRNAs like EIciEIF3J and EIciPAIP2 can strengthen transcription of parental genes [9]. EIciEIF3J and EIciPAIP2 were shown to intervene in the transcription of EIF3J and PAIP2 parent transcripts and lowered their levels in a cisacting fashion. Furthermore, as these EIciRNAs are not limited to their parental gene loci, it is likely that they may also have transeffects, perhaps altering the transcription of genes on distal loci [43]. This highlights their roles in promoting gene transcription, but in this particular case, the genes they upregulate, that is, Eukaryotic Translation Initiation Factor 3 Subunit J (EIF3J) and poly(A)-binding protein interacting protein 2 (PAIP2), also have roles in protein synthesis. This could imply that the respective circRNAs may indirectly alter protein expression.

Studies on backsplicing knockouts of circRNAs from the formin (Fmn) gene in mice provided evidence for the regulatory functions of circRNAs in transcription [44]. CDR1, a circRNA seen to cause cell proliferation in colorectal cancer [38], was identified as a post-transcriptional regulator by acting as an miR-7 sponge in brain tissues of mice and zebrafish, which impaired midbrain development in zebrafish [22]. The stronger regulatory function of a single exon compared to exon-intron and intron-intron was elucidated. This suggests that EcRNAs could act as "mRNA traps" by sequestering the translation start site, leading to the formation of dysfunctional transcripts [7].

\section{Protein Interactions}

The interactions of circRNAs with RBPs is another wellknown function. An instance of circRNA-protein interaction is that of circMbl binding to the muscleblind (MBL) protein which is a splicing factor known to promote circ$\mathrm{Mbl}$ production. This circRNA was suggested to be in-

Gastrointest Tumors 2021;8:159-168 DOI: $10.1159 / 000517303$ 
volved in the regulation of levels of MBL by sponging out excess MBL proteins. In fact, MBL was suggested to increase the generation of circMbl when MBL is in excess, in order to control its levels [14]. CircRNA-protein interactions have been shown to be important for post-transcriptional regulation and are also associated with cell cycle progression based on the discovery of the ternary complex circ-Foxo3-p21-CDK2 [45]. The involvement of the circRNA circ-Foxo3 in this regulatory network highlights its role in the regulation of increased cancer cell proliferation. Other circRNAs should also be assessed for this function.

\section{Translated CircRNAs}

Despite being known to be "non-coding" in nature, certain circRNAs possess internal ribosome entry site elements which enable translation of the circular molecule to generate high-molecular-weight polypeptides. This novel feature, attributed to circ-SHPRH, has been proven to be beneficial for activities like tumour suppression in glioblastomas $[45,46]$.

Synthetic exon-exon circRNAs have been shown to have protein-coding capacity; for example, Circ-FBXW7, an exon-exon circRNA, encodes a protein, FBXW7-185aa [47]. A recent study showed that the expression of hsa circ_0000423 (also known as circPPP1R12A) was significantly increased in colon cancer (CC) cells. These researchers further demonstrated that this circRNA encodes a conserved 73-aa peptide, PPP1R12A-C; this peptide itself, and not the circRNA, promoted the growth ofCC cells in vitro and in vivo. In addition, circPPP1R12A73aa, a functional protein encoded by the same circRNA, promoted the growth and metastasis of CC by activation of the Hippo-YAP signalling pathway [48]. Thus, some circRNA, including those that act as ceRNA, can be translated into biologically relevant peptides [49] such as those encoded by circRNAs with suppressive properties in glioma [50]. The biogenesis and functions of circRNAs are depicted in Figure 1.

\section{Significance of circRNA in GC}

The therapeutic potentials of circRNAs are beginning to emerge, based on the association of circRNA with diseases via its interaction with respective miRNAs associated with the respective disease. MiRNA-circRNA interactions are being evaluated by bioinformatic analyses [28-30] and Gene Ontology enrichment analyses to observe the enrichment of genes associated with particular biological processes [51].
The association of circRNAs with cancer-related miRNAs highlights the significance of circRNAs in cancer, and particularly GC. Therefore, these oncogenic and tumour-suppressing functions bring about alteration of the proliferation, invasion, and migration of GC cells. By their action on miRNAs, they influence downstream miRNA molecular signalling pathways including PTEN, CREB1, CDK6, NR4A2, NEK2, MAP7, AKT3, and miR$646 / C D 6$, among others [52, 53].

Several studies have demonstrated the miRNA-sponging capabilities of circRNA in GC; for example, the circRNA ciRS-7 is overexpressed and thus nullifies the effect of miR-7-induced tumour suppression via the PTEN/ PI3K/AKT signalling pathway [54]. This was linked to the promotion of proliferation, inhibition of apoptosis, and correlation between TNM stage and poor survival time. CircPVT1 is upregulated in GC and may promote cell proliferation by sponging members of the miR-irs 125 family [55]. Hsa_circ_0081143 was reported to promote the proliferation and invasion of GC cells; interestingly, this circRNA reduced the sensitivity of GC cells to cisplatin in vitro. In contrast, hsa_circ_0081143 was suppressed in vivo, along with reduced growth of GC cells and enhanced sensitivity to cisplatin. Further analysis revealed that this circRNA sponges and thus regulates miR646, which further interacts with CD6. The provocative suggestion is that hsa_circ_0081143 may play an oncogene-like role as a mediator of GC progression [53].

Expression profile analysis of circRNAs by highthroughput microarrays showed a significant difference between the expression of circRNAs in GC tissue and healthy non-tumour tissue. Among 308 circRNAs studied, $34.7 \%$ were upregulated while $65.26 \%$ were downregulated. Many of the circRNAs aberrantly expressed in GC are neither over- nor underexpressed in other cancer tissues, suggesting distinct expression patterns of circRNA in GC.For example, the circRNA, hsa_circ_0014717, was downregulated in $77.2 \%$ of GC tissues.

The same trend of downregulation of this circRNA was seen in chronic atrophic gastritis, a known risk factor of developing GC. Moreover, its levels correlated with tumour stage, distal metastasis, and expression of tissue carcinoembryonic antigen and carbohydrate antigen 19-9 which are also prognostic markers of GC. The ability of

Fig. 1. Biogenesis and functions of circRNAs. CircRNA, circular RNA; miRNA, microRNA; EcRNA, exonic circular RNA; EIciRNA, exon-intron circular RNA; ciRNA, intronic circular RNA; IRES, internal ribosome entry site; U1 snRNP, U1 small nuclear ribonucleoprotein particle; RNA POL II, RNA polymerase II.

(For figure see next page.)
Gastrointest Tumors 2021;8:159-168 DOI: $10.1159 / 000517303$
Rajgopal/Fredrick/Parvathi 


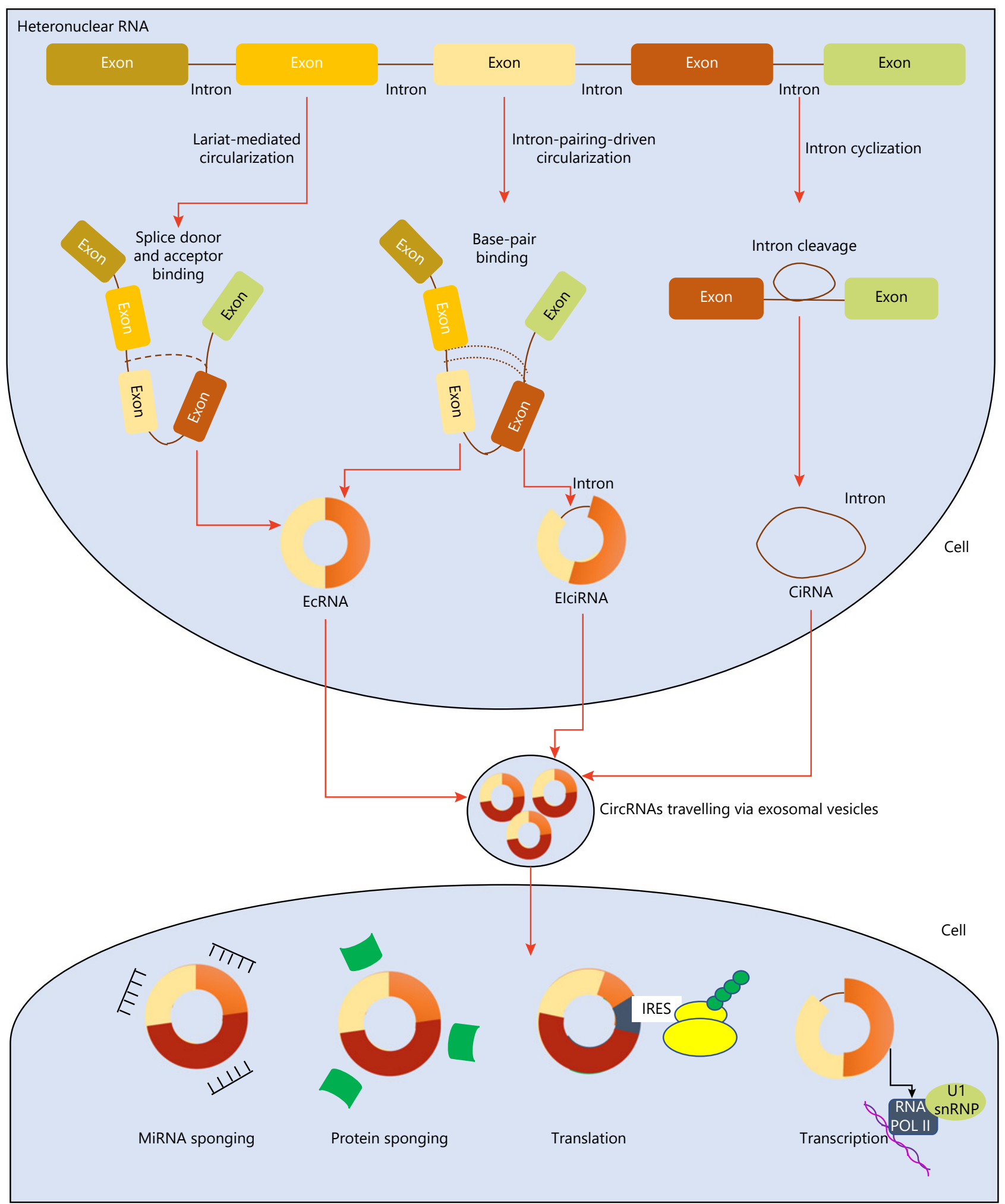

Legend: CircRNA: Circular RNA; MiRNA: Micro RNA; EcRNA: Exonic Circular RNA; ElcIRNA: Exon-Intron Circular RNA; CiRNA:

Intronic Circular RNA; IRES: Internal Ribosome Entry Site; U1 snRNP: U1small nuclear ribonucleoprotein particle; RNA POL II: RNA Polymerase II 
hsa_circ_0014717 to stably exist in gastric juice is also a significant finding, as it may hold promise as a biomarker for GC [56]. However, certain discrepancies do exist such as with the circRNA hsa_circ_0074362; it was reported to be one of the top 10 upregulated circRNAs in GC [56], but a more recent study claimed that the same circRNA is significantly downregulated in GC [57].

Other studies have demonstrated different sets of circRNAs to be upregulated or downregulated in the plasma and tissue of patients with GC [48, 49]. These aberrantly expressed circRNAs hold much promise as biomarkers in non-invasive tests. Therefore, they could play major roles in the prognosis and diagnosis of GC; this will be elaborated upon later in this review.

Although some circRNAs have been shown to promote tumourigenesis, some inhibit tumour development and metastasis. For example, circ_0027599 is significantly downregulated in GC tissues and interacts with miR-101 which regulates the GC suppressor gene pleckstrin homologous domain family member 1 ; computational prediction suggests that this interaction reduces the survival and metastasis of GC [58]. Circ_101057 has been shown to sponge miR-424-5p and thus acts as a tumour suppressor molecule, as this miRNA family governs the expression of large tumour suppressor kinase 1 (LATS1). Overexpression of miR-424-5p causes a decreased expression of LATS1, which stimulates proliferation and invasion in GC [51]. Another instance is that of tumour suppression by hsa_circ_0001368 by sponging miR-6506-5p whose target is forkhead box O3 (FOXO3), a tumour suppressor. Knockout of hsa_circ_0001368 resulted in decreased expression of FOXO3, indicating that this circRNA, by its interaction with miR-6506-5p and FOXO3, has tumour suppressive functions [59]. Other circRNAs that are associated with inhibition or promotion of cell proliferation, invasion, and distal and lymphatic metastasis are hsa_circ_00000 96 [60], hsa_circ_0000190 [61], hsa_circ_0000520 [62], hsa_circ_0000745 [63], hsa_circ_0000467 [64], and hsa_circ_0017639 [65].

CircRNAs have also been shown to be involved in the epithelial-mesenchymal transition (EMT), a vital aspect in the progression of cancer metastasis. EMT is associated with a decrease in E-cadherin and increase in vimentin in the tumour microenvironment. Circ104916 is reported to upregulate E-cadherin and downregulate $\mathrm{N}$-cadherin, vimentin, and Slug, propounding the involvement of circRNAs in EMT during tumourigenesis and in turn invasion and metastasis [66]. CircRNAs have been described as interacting with EMTtranscription factors such as Snail, Twist, Slug, and Zeb which regulate the process of EMT and EMT-related signalling pathways [67]. As previously mentioned, ciRS-7 acts on miR-7 which has a tumour suppressive role in GC. In other cancers, miR-7 has been reported to target IGF1R [68, 69] and FAK [70] and thus upregulate E-cadherin, resulting in a decrease in EMT and in turn suppression of metastasis [71]. This cascade can be ultimately traced back to the interaction of ciRS-7 with miR-7. Overexpression of ciR-7 can indirectly stimulate the process of EMT. However, much more research needs to be focused on the contribution of circRNAs in EMT in GC.

\section{Clinical Applications of circRNAs in GC}

Research focused on exosomal components and circRNAs has recognized their interdependent relations with human diseases. Recent studies have shown major involvement of circRNAs in colorectal cancer [72], oesophageal cancer [73], hepatic cancer [74], urinary bladder cancer [75], and oral squamous cell carcinoma [76], among others. They are responsible for modifying various pathways, including the mTOR pathway [77], NF$\kappa \mathrm{B} / \mathrm{p} 65$ pathway [78], and $\mathrm{Wnt} / \beta$-catenin pathway [79], likewise.

GC involves the development of malignant tumours in the gastric mucosal epithelium, mediated by several mechanisms mentioned earlier. These pathways differ from the typical behaviour, creating abnormal effects on the downstream targets. CircRNAs are key regulatory factors involved in several pathways.

The expression levels of the GC-related circRNAs are shown to alter with the state of the cell and the stages of the disease progression, as seen in circRNA_100269 [44]. CircRNA_0000190 [40], circRNA_0000520 [62], circRNA_LARP4 [7], etc., have been verified to be underexpressed in the affected tissues in comparison with the adjacent normal tissues. Hence, comparative analysis between tumour environment and the normal tissue can improve prognostic abilities.

The stable expression of circRNAs has enabled them to be present in gastric juice and plasma, as in the case of circRNA_0014717 [56], circRNA_0001017 [80], circRNA_0061276 [80], and circRNA_0000745 [63]. The documented correlation between tissue and plasma levels of circRNAs establishes them as favourable blood-based biomarkers for GC.

The major contributions of circRNAs were found to occur in the regulation of proliferation, migration, and 
metastasis. Analysis by RT-qPCR and bioinformatic tools highlighted that the expression levels of circPVRL3 [81], circRNA_0000096 [60], circRNA_100269 [44], circRNA_0014717 [56], and circPVT1 [73] varied in accordance with the processes described above. A major challenge in oncology today is the prediction of the rate of growth and metastasis. With circRNAs as biomarkers, such processes can be tracked in order to generate better diagnosis and prognosis. Certain circRNAs have proven to have tumour suppressor effects. Such activity was observed particularly in circRNA LARP4 [82] and circRNA-104916 [83] among others. In conclusion, the benefits of utilizing circRNAs are its non-invasive nature and specificity, aiding the early detection of GC and predictions of the tumour growth and metastasis.

\section{Future Perspectives}

The fascinating network of circRNAs, their behaviour as ceRNAs, and communication with miRNAs are extremely complex. In addition, the high number of various structures of circRNAs makes detection and identification of their roles very challenging. Numerous aspects of circRNAs await elucidation; these include their biogenesis, cellular location, regulatory mechanisms, and pathological consequences. Nonetheless, there is much scope for research on circRNAs that will lead to better prognosis and earlier diagnosis of GC, including possible management of GC. Appropriate upregulation and/or downregulation of relevant circRNAs could help manipulate the invasion or metastatic ability of GC cells, thus improving the survival rate of patients with GC.

Cachexia, a major manifestation of cancer, is characterized by excessive wastage of muscle and fat, indicating a very severe stage of the disease. One of the contributing factors to energy wasting in cachexia is the conversion of white adipose tissue (WAT) to brown adipose tissue. This has been attributed to the loss of PRDM16, a transcriptional coregulator which facilitates the browning process $[84,85]$. Another notable mediator of brown adipose tissue is uncoupling protein 1 . The browning of WAT has been shown to occur to a 3 times greater extent in patients with GC. Exosome delivery of circRNAs ciRS-133 derived from GC cells has been demonstrated to promote the browning of WAT by suppressing miR-133 and thus upregulating PRDM16. Inhibition of exosome generation and silencing of ciRS-133 expression has been proposed as a strategy for preventing cancer-related cachexia and possibly for increasing the survival time of patients with GC [86].

The proposed function of circRNAs as regulators of the EMT pathway and metastasis is also worthy of consideration. Identifying circRNAs that network with EMTrelated signalling pathways holds promise as in the case of circ-104916 in GC [83] and circPTK2 in non-small cell lung cancer [87]. EMT-related circRNAs could well be considered as potential biomarkers and for therapeutic targeting.

The unravelling of these precise molecular mechanisms is likely to lead to therapeutic regimens and even to individualized treatment. Pre-requisites include a better understanding of the expression of novel circRNAs, their binding targets, and downstream processes using techniques such as RNA immunoprecipitation combined with luciferase reporter assays [88] and bioinformatic and prediction studies. Computational tools such as StarBase, Circinteractome, Circ2Traits, and CLIP-seq [29, 51, 89, 90] as well as RNA-seq datasets and algorithms (CircRNA-finder, Find-circ, and CIRCexplorer) can help in the detection of circRNAs. RT-qPCR is quite promising in this regard, though it is associated with false positives and bias [91]. Other molecular techniques that show great promise in the field of circRNA-related cancer research include Northern blotting (to circumvent amplificationinduced problems in RT-qPCR) and fluorescence in situ hybridization for spatial localization of circRNAs [92, 93].

Strategies for targeting circRNAs for therapy include the induction of tumour suppressive activity via circRNA or the alteration of their oncogenic roles by interfering with backsplicing mechanisms [91]. For example, hsa circ_0081143 appears to play an oncogenic role in the progression of GC, as well as resistance to cisplatin; this suggests the possibility of therapeutically targeting it, particularly because its suppression revealed promising results in terms of decreased cell growth and increased sensitivity to cisplatin [53]. Nanobiology may prove to be useful in the delivery of circRNAs; a recent study used circ-Foxo3 plasmids conjugated with gold nanoparticles to induce tumour apoptosis via circ-Foxo3 [94].

The fascinating leads described in this review suggest numerous opportunities for clinical applications based on the physiological and pathological functions of circRNAs. Their functional role in tumorigenesis is just starting to be elucidated. What we have understood about circRNAs makes them promising candidates not just as valuable biomarkers for cancer but also as potential targets in cancer therapy. 


\section{Conclusion}

CircRNAs offer much promise for the improvement of diagnosis, prognosis, and possible targeted treatment of GC, given their impact on the cancer-related pathological events of proliferation, invasion, and migration. They are additionally associated with clinicopathological features of differentiation, TNM staging, and metastasis. Further research is awaited concerning its exact mechanisms in these events. The use of bioinformatic analysis tools will also play a big role in further understanding these mechanisms. At present, the most promising outcomes of research lie in its usage as biomarkers for the early detection and prognosis of GC.

\section{Conflict of Interest Statement}

The authors have no conflicts of interest to declare.

\section{Funding Sources}

No funding was received for this study.

\section{Author Contributions}

S.R., S.J.F., and V.D.P. have read and agreed to the final version of the manuscript. S.R. and S.J.F. developed the review concept and wrote the manuscript. S.R., S.J.F., and V.D.P. designed and constructed the diagram and flow of information. S.R., S.J.F., and V.D.P. reviewed and edited the manuscript and approved the final submission.

\section{References}

1 Torre LA, Bray F, Siegel RL, Ferlay J, LortetTieulent J, Jemal A. Global cancer statistics, 2012. CA Cancer J Clin. 2015 Mar;65(2):87108.

2 Sanger HL, Klotz G, Riesner D, Gross HJ, Kleinschmidt AK. Viroids are single-stranded covalently closed circular RNA molecules existing as highly base-paired rod-like structures. Proc Natl Acad Sci U S A. 1976;73(11): $3852-6$.

3 Hansen TB, Wiklund ED, Bramsen JB, Villadsen SB, Statham AL, Clark SJ, et al. MiRNAdependent gene silencing involving Ago2-mediated cleavage of a circular antisense RNA. EMBO J. 2011 Nov;30(21):4414-22.

4 Al-Balool HH, Weber D, Liu Y, Wade M, Guleria K, Nam PL, et al. Post-transcriptional exon shuffling events in humans can be evolutionarily conserved and abundant. Genome Res. 2011 Nov;21(11):1788-99.

5 Kelly S, Greenman C, Cook PR, Papantonis A. Exon skipping is correlated with exon circularization. J Mol Biol. 2015 Jul;427(15):2414-7.

6 Vicens Q, Westhof E. Biogenesis of circular RNAs. Cell. 2014 Sep;159(1):13-4.

7 Jeck WR, Sharpless NE. Detecting and characterizing circular RNAs. Nat Biotechnol. 2014;32(5):453-61.

8 Shan C, Zhang Y, Hao X, Gao J, Chen X, Wang K. Biogenesis, functions and clinical significance of circRNAs in gastric cancer. Mol Cancer. 2019 Sep;18(1):136.

9 Li Z, Huang C, Bao C, Chen L, Lin M, Wang $\mathrm{X}$, et al. Exon-intron circular RNAs regulate transcription in the nucleus. Nat Struct Mol Biol. 2015 Mar;22(3):256-64.

10 Senfter D, Holzner S, Kalipciyan M, Staribacher A, Walzl A, Huttary N, et al. Loss of miR-200 family in 5-fluorouracil resistant colon cancer drives lymphendothelial invasiveness in vitro. Hum Mol Genet. 2015 Jul; 24(13):3689-98.
11 Zhang Y, Zhang XO, Chen T, Xiang JF, Yin QF, Xing YH, et al. Circular intronic long noncoding RNAs. Mol Cell. 2013 Sep;51(6): 792-806.

12 Ivanov A, Memczak S, Wyler E, Torti F, Porath $\mathrm{HT}$, Orejuela MR, et al. Analysis of intron sequences reveals hallmarks of circular RNA biogenesis in animals. Cell Rep. 2015 Jan; 10(2):170-7.

13 Conn SJ, Pillman KA, Toubia J, Conn VM, Salmanidis M, Phillips CA, et al. The RNA binding protein quaking regulates formation of circRNAs. Cell. 2015 Mar;160(6): 1125-34.

14 Ashwal-Fluss R, Meyer M, Pamudurti NR, Ivanov A, Bartok O, Hanan M, et al. CircRNA Biogenesis competes with pre-mRNA splicing. Mol Cell. 2014;56(1):55-66.

15 Zhang Y, Xue W, Li X, Zhang J, Chen S, Zhang $\mathrm{JL}$, et al. The biogenesis of nascent circular RNAs. Cell Rep. 2016 Apr;15(3):611-24.

16 Li Y, Zheng Q, Bao C, Li S, Guo W, Zhao J, et al. Circular RNA is enriched and stable in exosomes: a promising biomarker for cancer diagnosis. Cell Res. 2015 Aug;25(8):981-4.

17 Salzman J, Gawad C, Wang PL, Lacayo N, Brown PO. Circular RNAs are the predominant transcript isoform from hundreds of human genes in diverse cell types. PLoS One. 2012 Feb;7(2):e30733.

18 Jeck WR, Sorrentino JA, Wang K, Slevin MK, Burd CE, Liu J, et al. Circular RNAs are abundant, conserved, and associated with ALU repeats. RNA. 2013 Feb;19(2):141-57.

19 Suzuki H, Tsukahara T. A view of pre-mRNA splicing from RNase $\mathrm{R}$ resistant RNAs. Int J Mol Sci. 2014 May;15(6):9331-42.

20 Fang X, Wen J, Sun M, Yuan Y, Xu Q. CircRNAs and its relationship with gastric cancer. J Cancer. 2019;10(24):6105-13.

21 AbouHaidar MG, Venkataraman S, Golshani A, Liu B, Ahmad T. Novel coding, translation, and gene expression of a replicating covalently closed circular RNA of 220 nt. Proc Natl Acad Sci U S A. 2014 Oct;111(40):14542-7.

22 Memczak S, Jens M, Elefsinioti A, Torti F, Krueger J, Rybak A, et al. Circular RNAs are a large class of animal RNAs with regulatory potency. Nature. 2013 Mar;495(7441):333-8.

23 Salzman J, Chen RE, Olsen MN, Wang PL, Brown PO. Cell-type specific features of circular RNA expression. PLoS Genet. 2013 Sep; 9(9):e1003777.

24 Liang G, Yang Y, Niu G, Tang Z, Li K. Genomewide profiling of sus scrofa circular RNAs across nine organs and three developmental stages. DNA Res. 2017 Oct;24(5):523-35.

25 Lasda E, Parker R. Circular RNAs co-precipitate with extracellular vesicles: a possible mechanism for circRNA clearance. PLoS One. 2016 Feb;11(2):e0148407.

26 Wang Y, Wang Z. Efficient backsplicing produces translatable circular mRNAs. RNA. 2015 Feb;21(2):172-9.

27 Jiang F, Shen X. Current prevalence status of gastric cancer and recent studies on the roles of circular RNAs and methods used to investigate circular RNAs. Cell Mol Biol Lett. 2019 Aug;24(1):53.

28 Panda AC. Circular RNAs act as miRNA sponges. Adv Exp Med Biol. 2018;1087:67-79.

29 Li JH, Liu S, Zhou H, Qu LH, Yang JH. StarBase v2.0: decoding miRNA-ceRNA, miRNA-ncRNA and protein-RNA interaction networks from large-scale CLIP-seq data. $\mathrm{Nu}$ cleic Acids Res. 2014 Jan;42(D1):D92.

30 Hansen TB, Jensen TI, Clausen BH, Bramsen JB, Finsen B, Damgaard CK, et al. Natural RNA circles function as efficient microRNA sponges. Nature. 2013 Mar;495(7441):384-8.

$31 \mathrm{Xu} \mathrm{H}$, Guo S, Li W, Yu P. The circular RNA Cdrlas, via miR-7 and its targets, regulates insulin transcription and secretion in islet cells. Sci Rep. 2015 Jul;5:12453. 
32 Zheng Q, Bao C, Guo W, Li S, Chen J, Chen $\mathrm{B}$, et al. Circular RNA profiling reveals an abundant circHIPK3 that regulates cell growth by sponging multiple miRNAs. Nat Commun. 2016 Apr;7(1):11215-3.

33 Tian F, Wang Y, Xiao Z, Zhu X. [Circular RNA CircHIPK3 promotes NCI-H1299 and NCI-H2170 cell proliferation through miR379 and its target IGF1]. Zhongguo Fei Ai Za Zhi. 2017 Jul;20(7):459-67.

34 Hsiao KY, Lin YC, Gupta SK, Chang N, Yen L, Sun HS, et al. Noncoding effects of circular RNA CCDC66 promote colon cancer growth and metastasis. Cancer Res. 2017 May;77(9): 2339-50.

35 Bachmayr-Heyda A, Reiner AT, Auer K, Sukhbaatar N, Aust S, Bachleitner-Hofmann $\mathrm{T}$, et al. Correlation of circular RNA abundance with proliferation: exemplified with colorectal and ovarian cancer, idiopathic lung fibrosis, and normal human tissues. Sci Rep. 2015;5:8057.

36 Zhong Y, Du Y, Yang X, Mo Y, Fan C, Xiong $F$, et al. Circular RNAs function as ceRNAs to regulate and control human cancer progression. Mol Cancer. 2018 Apr;17(1):79.

37 Wang K, Long B, Liu F, Wang JX, Liu CY, Zhao B, et al. A circular RNA protects the heart from pathological hypertrophy and heart failure by targeting miR-223. Eur Heart J. 2016 Sep;37(33):2602-11.

38 Tang W, Ji M, He G, Yang L, Niu Z, Jian M, et al. Silencing CDR1as inhibits colorectal cancer progression through regulating microRNA-7. Onco Targets Ther. 2017 Apr;10:2045-56.

39 Tang YY, Zhao P, Zou TN, Duan JJ, Zhi R, YangSY, etal.CircularRNAhsa_circ_0001982 promotes breast cancer cell carcinogenesis through decreasing miR-143. DNA Cell Biol. 2017 Nov;36(11):901-8.

40 Lasda E, Parker R. Circular RNAs: diversity of form and function. RNA. 2014 Dec;20(12): 1829-42.

41 Liu J, Liu T, Wang X, He A. Circles reshaping the RNA world: from waste to treasure. $\mathrm{Mol}$ Cancer. 2017 Mar; 16(1):58.

42 Kelly S, Greenman C, Cook PR, Papantonis A. Exon skipping is correlated with exon circularization. J Mol Biol. 2015 Jul;427(15):2414-7.

43 Bose R, Ain R. Regulation of transcription by circular RNAs. Adv Exp Med Biol. 2018;1087: 81-94.

44 Chao CW, Chan DC, Kuo A, Leder P. The mouse formin (Fmn) gene: abundant circular RNA transcripts and gene-targeted deletion analysis. Mol Med. 1998;4(9):614-28.

45 Du WW, Yang W, Liu E, Yang Z, Dhaliwal P, Yang BB. Foxo3 circular RNA retards cell cycle progression via forming ternary complexes with p21 and CDK2. Nucleic Acids Res. 2016 Feb;44(6):2846-58.

46 Chen CY, Sarnow P. Initiation of protein synthesis by the eukaryotic translational apparatus on circular RNAs. Science. 1995; 268(5209):415-7.

47 Yang Y, Gao X, Zhang M, Yan S, Sun C, Xiao $F$, et al. Novel role of FBXW7 circular RNA in repressing glioma tumorigenesis. J Natl Cancer Inst. 2018 Mar;110(3):304-15.

48 Zheng X, Chen L, Zhou Y, Wang Q, Zheng Z, $\mathrm{Xu} \mathrm{B}$, et al. A novel protein encoded by a circular RNA circPPP1R12A promotes tumor pathogenesis and metastasis of colon cancer via Hippo-YAP signaling. Mol Cancer. 2019 Mar;18(1):47.

49 Matsumoto A, Nakayama KI. Hidden peptides encoded by putative noncoding RNAs. Cell Struct Funct. 2018;43(1):75-83.

50 Zhang M, Huang N, Yang X, Luo J, Yan S, Xiao F, et al. A novel protein encoded by the circular form of the SHPRH gene suppresses glioma tumorigenesis. Oncogene. 2018 Mar; 37(13):1805-14.

51 Ghosal S, Das S, Sen R, Basak P, Chakrabarti J. Circ2Traits: a comprehensive database for circular RNA potentially associated with disease and traits. Front Genet. 2013 Dec; $4: 283$

52 Wang KW, Dong M. Role of circular RNAs in gastric cancer: recent advances and prospects. World J Gastrointest Oncol. 2019;11(6):45969.

53 Xue M, Li G, Fang X, Wang L, Jin Y, Zhou Q. hsa_circ_0081143 promotes cisplatin resistance in gastric cancer by targeting miR-646/ CDK6 pathway. Cancer Cell Int. 2019 Feb; 19(1):25.

54 Pan H, Li T, Jiang Y, Pan C, Ding Y, Huang Z, et al. Overexpression of circular RNA ciRS-7 abrogates the tumor suppressive effect of miR-7 on gastric cancer via PTEN/PI3K/AKT signaling pathway. J Cell Biochem. 2018 Jan; 119(1):440-6.

55 Chen J, Li Y, Zheng Q, Bao C, He J, Chen B, et al. Circular RNA profile identifies circPVT1 as a proliferative factor and prognostic marker in gastric cancer. Cancer Lett. 2017 Mar;388:208-19.

56 Shao Y, Li J, Lu R, Li T, Yang Y, Xiao B, et al. Global circular RNA expression profile of human gastric cancer and its clinical significance. Cancer Med. 2017 Jun;6(6):1173-80.

57 Xie Y, Shao Y, Sun W, Ye G, Zhang X, Xiao B, et al. Downregulated expression of hsa circ_0074362 in gastric cancer and its potential diagnostic values. Biomark Med. 2018 Jan; 12(1):11-20.

58 Wang L, Shen J, Jiang Y. Circ-0027599/PHDLA1 suppresses gastric cancer progression by sponging miR-101-3p.1 11 medical and health sciences 1112 oncology and carcinogenesis 06 biological sciences 0601 biochemistry and cell biology. Cell Biosci. 2018 Nov; $8(1): 58$.

59 Lu J, Zhang PY, Li P, Xie JW, Wang JB, Lin JX, et al. Circular RNA hsa_circ_0001368 suppresses the progression of gastric cancer by regulating miR-6506-5p/FOXO3 axis. Biochem Biophys Res Commun. 2019 Apr; 512(1):29-33

60 Li P, Chen H, Chen S, Mo X, Li T, Xiao B, et al. Circular RNA 0000096 affects cell growth and migration in gastric cancer. Br J Cancer. $2017 \mathrm{Feb} ; 116(5): 626-33$.
61 Chen S, Li T, Zhao Q, Xiao B, Guo J. Using circular RNA hsa_circ_0000190 as a new biomarker in the diagnosis of gastric cancer. Clin Chim Acta. 2017 Mar;466:167-71.

62 Sun H, Tang W, Rong D, Jin H, Fu K, Zhang W, et al. Hsa_circ_0000520, a potential new circular RNA biomarker, is involved in gastric carcinoma. Cancer Biomark. 2018 Feb;21(2): 299-306.

63 Huang M, He YR, Liang LC, Huang Q, Zhu ZQ. Circular RNA hsa_circ_0000745 may serve as a diagnostic marker for gastric cancer. World J Gastroenterol. 2017 Sep;23(34):6330-8.

64 Lu J, Zhang PY, Xie JW, Wang JB, Lin JX, Chen Q, et al. Hsa_circ_0000467 promotes cancer progression and serves as a diagnostic and prognostic biomarker for gastric cancer. J Clin Lab Anal. 2019 Mar;33(3):e22726.

65 Sun H, Xi P, Sun Z, Wang Q, Zhu B, Zhou J, et al. Circ-SFMBT2 promotes the proliferation of gastric cancer cells through sponging miR-182-5p to enhance CREB1 expression. Cancer Manag Res. 2018;10:5725-34.

66 Li J, Zhen L, Zhang Y, Zhao L, Liu H, Cai D, et al. Circ-104916 is downregulated in gastric cancer and suppresses migration and invasion of gastric cancer cells. Onco Targets Ther. 2017 Jul; 10:3521-9.

67 Shang BQ, Li ML, Quan HY, Hou PF, Li ZW, Chu SF, et al. Functional roles of circular RNAs during epithelial-to-mesenchymal transition. Mol Cancer. 2019 Sep;18(1):138.

68 Zhao X, Dou W, He L, Liang S, Tie J, Liu C, et al. MicroRNA-7 functions as an anti-metastatic microRNA in gastric cancer by targeting insulin-like growth factor-1 receptor. Oncogene. 2013 Mar;32(11):1363-72.

69 Jiang L, Liu X, Chen Z, Jin Y, Heidbreder CE, Kolokythas A, et al. MicroRNA-7 targets IGF1R (insulin-like growth factor 1 receptor) in tongue squamous cell carcinoma cells. Biochem J. 2010;432(1):199-205.

70 Kong X, Li G, Yuan Y, He Y, Wu X, Zhang W, et al. MicroRNA-7 inhibits epithelial-to-mesenchymal transition and metastasis of breast cancer cells via targeting FAK expression. PLoS One. 2012 Aug;7(8):e41523.

71 Li J, Yang J, Zhou P, Le Y, Zhou C, Wang S, et al. Circular RNAs in cancer: novel insights into origins, properties, functions and implications. Am J Cancer Res. 2015 [cited 2020 Sep 8;5(2):472-80.

72 Xie H, Ren X, Xin S, Lan X, Lu G, Lin Y, et al. Emerging roles of circRNA_001569 targeting miR-145 in the proliferation and invasion of colorectal cancer. Oncotarget. 2016 May; 7(18):26680-91.

73 Li F, Zhang L, Li W, Deng J, Zheng J, An M, et al. Circular RNA ITCH has inhibitory effect on ESCC by suppressing the Wnt/ $\beta$-catenin pathway. Oncotarget. 2015;6(8):6001-13.

74 Zhang H, Deng T, Ge S, Liu Y, Bai M, Zhu K, et al. Exosome circRNA secreted from adipocytes promotes the growth of hepatocellular carcinoma by targeting deubiquitination-related USP7. Oncogene. 2019 Apr;38(15): 2844-59. 
75 Lu Q, Liu T, Feng H, Yang R, Zhao X, Chen $\mathrm{W}$, et al. Circular RNA circSLC8A1 acts as a sponge of miR-130b/miR-494 in suppressing bladder cancer progression via regulating PTEN. Mol Cancer. 2019 Dec;18(1):111.

76 Chen L, Zhang S, Wu J, Cui J, Zhong L, Zeng L, et al. circRNA_100290 plays a role in oral cancer by functioning as a sponge of the miR29 family. Oncogene. 2017 Aug;36(32):455161.

77 Fang Y, Xue JL, Shen Q, Chen J, Tian L. MicroRNA-7 inhibits tumor growth and metastasis by targeting the phosphoinositide 3-kinase/Akt pathway in hepatocellular carcinoma. Hepatology. 2012;55(6):1852-62.

78 Li R, Ke S, Meng F, Lu J, Zou X, He Z, et al. CiRS-7 promotes growth and metastasis of esophageal squamous cell carcinoma via regulation of miR-7/HOXB13. Cell Death Dis. 2018 Aug;9(8):838.

79 Huang G, Zhu H, Shi Y, Wu W, Cai H, Chen $X$. Cir-ITCH plays an inhibitory role in colorectal cancer by regulating the $\mathrm{Wnt} / \beta$ catenin pathway. PLoS One. 2015 Jun;10(6) e0131225.

80 Li T, Shao Y, Fu L, Xie Y, Zhu L, Sun W, et al. Plasma circular RNA profiling of patients with gastric cancer and their droplet digital RT-PCR detection. J Mol Med. 2018 Jan; 96(1):85-96.
81 Sun HD, Xu ZP, Sun ZQ, Zhu B, Wang Q, Zhou J, et al. Down-regulation of circPVRL3 promotes the proliferation and migration of gastric cancer cells. Sci Rep. 2018 Dec;8(1): 10111.

82 Zhang J, Liu H, Hou L, Wang G, Zhang R, Huang Y, et al. Circular RNA_LARP4 inhibits cell proliferation and invasion of gastric cancer by sponging miR-424-5p and regulating LATS1 expression. Mol Cancer. 2017 Sep; 16(1):151.

83 Li J, Zhen L, Zhang Y, Zhao L, Liu H, Cai D, et al. Circ-104916 is downregulated in gastric cancer and suppresses migration and invasion of gastric cancer cells. Onco Targets Ther. 2017 Jul; 10:3521-9.

84 Kir S, Spiegelman BM. Cachexia and brown fat: a burning issue in cancer. Trends Cancer. 2016 Sep;2(9):461-3.

85 Cohen P, Levy JD, Zhang Y, Frontini A, Kolodin DP, Svensson KJ, et al. Ablation of PRDM16 and beige adipose causes metabolic dysfunction and a subcutaneous to visceral fat switch. Cell. 2014;156(1-2):304-16.

86 Zhang H, Zhu L, Bai M, Liu Y, Zhan Y, Deng $\mathrm{T}$, et al. Exosomal circRNA derived from gastric tumor promotes white adipose browning by targeting the miR-133/PRDM16 pathway. Int J Cancer. 2019 May;144(10):2501-15.
87 Wang L, Tong X, Zhou Z, Wang S, Lei Z, Zhang T, et al. Circular RNA hsa-circ-0008305 (circPTK2) inhibits TGF- $\beta$-induced epithelial-mesenchymal transition and metastasis by controlling TIF1 $\gamma$ in non-small cell lung cancer. Mol Cancer. 2018 Sep;17(1):40.

88 Li Y, Chen B, Huang S. Identification of circRNAs for miRNA targets by Argonaute2 RNA immunoprecipitation and luciferase screening assays. Methods Mol Biol. 2018; 1724:209-18.

89 Dudekula DB, Panda AC, Grammatikakis I, De S, Abdelmohsen K, Gorospe M. Circinteractome: a web tool for exploring circular RNAs and their interacting proteins and microRNAs. RNA Biol. 2016 Jan;13(1):34-42.

90 Zhang XQ, Yang JH. Discovering circRNAmicroRNA interactions from CLIP-seq data. Methods Mol Biol. 2018;1724:193-207.

91 Kristensen LS, Hansen TB, Venø MT, Kjems J. Circular RNAs in cancer: opportunities and challenges in the field. Oncogene. $2018 \mathrm{Feb}$; 37(5):555-65.

92 Zhang M, Xin Y. Circular RNAs: a new frontier for cancer diagnosis and therapy. J Hematol Oncol. 2018 Feb;11(1):21.

93 Zirkel A, Papantonis A. Detecting circular RNAs by RNA fluorescence in situ hybridization. Methods Mol Biol. 2018;172:69-75.

94 Du WW, Fang L, Yang W, Wu N, Awan FM, Yang Z, et al. Induction of tumor apoptosis through a circular RNA enhancing Foxo3 activity. Cell Death Differ. 2017 Feb;24(2):35770. 